\title{
Stellar models with the ML2 theory of convection (Research Note)
}

\author{
M. Salaris ${ }^{1}$ and S. Cassisi ${ }^{2}$ \\ 1 Astrophysics Research Institute, Liverpool John Moores University, Twelve Quays House, \\ Egerton Wharf Birkenhead CH41 1LD, UK \\ e-mail: ms@astro.livjm.ac.uk \\ 2 INAF - Osservatorio Astronomico di Collurania, via Mentore Maggini, 64100 Teramo, Italy \\ e-mail: cassisi@oa-teramo.inaf.it
}

Received 23 May 2008 / Accepted 17 June 2008

\section{ABSTRACT}

\begin{abstract}
Context. The mixing length theory (MLT) used to compute the temperature gradient in superadiabatic layers of stellar (interior and atmosphere) models contains in its standard form 4 free parameters. Three parameters are fixed a priori (and define what we denote as the MLT "flavour") whereas one (the so-called mixing length) is calibrated by reproducing observational constraints. The "classical" Böhm-Vitense flavour is used in all modern MLT-based stellar model computations and, despite its crude approximations, the resulting $T_{\text {eff }}$ scale appears - perhaps surprisingly - remarkably realistic, once the mixing length parameter is calibrated with a solar model. Aims. Model atmosphere computations employ parameter choices different from what is used in stellar interior modelling, raising the question of whether a single MLT flavour and mixing length value can be used to compute interiors and atmospheres of stars of all types. As a first step towards addressing this issue, we study whether the MLT flavour (the so-called ML2) and mixing length choice that have been proven adequate to model white dwarf atmospheres, are able to provide, when used in stellar models, results at least comparable to the use of the "classical" Böhm-Vitense flavour.

Methods. We have computed solar models and evolutionary tracks for both low- and intermediate-mass Population I and II stars, adopting both solar calibrated Böhm-Vitense and ML2 flavours of the MLT in our stellar evolution code, and state-of-the-art input physics.

Results. The two sets of models provide consistent results, with only minor differences. Both calibrations reproduce also the $T_{\text {eff }}$ of red giants in a sample of Galactic globular clusters. The ML2 solar model provides a mixing length about half the value of the local pressure scale height, thus alleviating - but not eliminating - one of the well known inconsistencies of the MLT employed in stellar models. This mixing length is also consistent with the value used in white dwarf model atmosphere computations.
\end{abstract}

Key words. convection - globular clusters: general - stars: interiors - stars: evolution - Sun: general - turbulence

\section{Introduction}

The equations still largely employed to determine the value of the temperature gradient in the superadiabatic regions of stellar convective envelopes and atmospheres are based on the so called "mixing length theory" (MLT). The MLT in its original form is a simple, local, time independent model, firstly applied to stellar modelling by Biermann (1932). Its description of convective transport considers the motion of "average" convective cells, all with the same physical properties at a given radial location $r$ within the star. These convective cells have a mean size $l\left(l=\alpha_{\mathrm{MLT}} H_{\mathrm{P}}\right.$ is the so-called "mixing length", where $\alpha_{\mathrm{MLT}}$ is a free parameter and $H_{\mathrm{P}}$ is the local pressure scale height $H_{\mathrm{P}} \equiv P / g \rho$ ) also equal to their mean free path, and an average convective speed $v_{\mathrm{c}}$ at a given value of $r$. There is in principle no reason why $\alpha_{\text {MLT }}$ should be kept constant when considering stars of different masses and/or chemical composition and/or at different evolutionary stages; even within the same star $\alpha_{\text {MLT }}$ might in principle vary from layer to layer, although stellar models usually keep $\alpha_{\text {MLT }}$ constant throughout the convective zone (but see Pedersen et al. 1990, for some low-mass stellar models computed with varying $\alpha_{\mathrm{MLT}}$ within the convective envelope). Keeping $\alpha_{\text {MLT }}$ constant still means a change of the cells' mean size and free path due to the variation of $H_{\mathrm{p}}$.
The value of $\alpha_{\mathrm{MLT}}$ is usually calibrated by reproducing some empirical constraint. In case of stellar models, it is typically the calibration of a theoretical solar model that determines the value of $\alpha_{\text {MLT. }}$ In all major stellar model databases (e.g. Girardi et al. 2000; VandenBerg et al. 2000; Yi et al. 2001; Pietrinferni et al. 2004; Dotter et al. 2007) this solar calibrated $\alpha_{\text {MLT }}$ is kept fixed in all evolutionary calculations of stars of different masses and chemical compositions. There are 3 additional free parameters entering the MLT that are generally fixed a priori, before the calibration of $\alpha_{\text {MLT }}$. They will be denoted here as $a, b, c$ following the formalism of Tassoul et al. (1990). These three parameters plus $\alpha_{\text {MLT }}$ enter the equations that determine $v_{\mathrm{c}}$, the average convective flux $F_{\mathrm{c}}$ and the convective efficiency $\Gamma$ (defined as the ratio between the excess heat content of a raising convective bubble just before its dissolution, and the energy radiated during its lifetime) at each point within the convective envelope. Once $a, b, c$ are fixed, the temperature gradient at a given location in the convective envelope of a stellar model and the resulting $T_{\text {eff }}$, depend on the value of $\alpha_{\text {MLT }}$. On the other hand, when $\alpha_{\text {MLT }}$ is kept fixed and one or more of the other three parameters are varied, the temperature gradients and $T_{\text {eff }}$ are also affected (e.g. Henyey et al. 1965).

The "classical" formulation of the MLT by Böhm-Vitense (1958) that is nowadays almost universally used in theoretical 
computations of stellar evolution models (denoted here as the BV58 "flavour" of the MLT) employs a specific set of choices for the values for $a, b$ and $c$. Solar calibrations with the present generation of stellar input physics provide a value of $\alpha_{\text {MLT }}$ typically around 2.0 (e.g. VandenBerg et al. 2000; Pietrinferni et al. 2004; Bahcall et al. 2006; Dotter et al. 2007; Morel \& Lebreton 2008) the precise value (for a given set of input physics) being dependent on the choice for the boundary conditions at the model surface.

The situation for model atmosphere computations is different. The model grids perhaps most used to produce synthetic spectra and bolometric corrections for evolutionary phases before the White Dwarf (WD) stage are the ATLAS9, MARCS and PHOENIX ones. The recent ATLAS9 grid described in Pietrinferni et al. (2004) employs $\alpha_{\mathrm{MLT}}=1.25$, while the latest MARCS models (Gustafsson et al. 2008) adopt a different value $\alpha_{\text {MLT }}=1.5$. There are some differences between the MLT flavours used in thse two sets of models, and also compared to the BV58 formalism used in stellar evolution computations (see Castelli et al. 1997; Gustafsson et al. 2008). ATLAS9 models with a much smaller $\alpha_{\mathrm{MLT}}=0.5$ have also been computed (Heiter et al. 2002). The recent Brott \& Hauschildt (2005) PHOENIX grid employs $\alpha_{\mathrm{MLT}}=2.0$ and (as communicated to us by our referee) the BV58 flavour of the MLT.

Yet another flavour of the MLT is employed in calculations of model atmospheres, spectra and bolometric corrections for WD stars (e.g. Bergeron et al. 1995a; Rohrmann 2001). It is the so-called ML2 (Tassoul et al. 1990), with its own specific choice of $a, b$ and $c$, different from the case of BV58. Bergeron et al. (1995b) have shown how for the case of ZZ Ceti stars, the ML2 with $\alpha_{\text {MLT }}=0.6$ provides overall consistency between temperature estimates from both UV and optical spectrum, observed photometry, gravitational redshift mass estimates and trigonometric parallax. The exact form of the MLT employed in WD interior models seem to be irrelevant (see, e.g. Fontaine et al. 2001) so that effectively one can employ the ML2 in computations of both convective atmospheres and envelopes of WDs.

This variety of choices regarding the 4 free parameters of the MLT should probably not be surprising. As already stated before, given the very approximate nature of the MLT, one cannot a priori expect the same choice of $\alpha_{\mathrm{MLT}}-$ and possibly also of $a, b$ and $c-$ to be appropriate for all evolutionary phases in both convective envelope and atmosphere of stars. It is however interesting, if only for heuristic purposes, to investigate whether a single flavour of the MLT with just one choice (or a small range of values) of $\alpha_{\mathrm{MLT}}$ is adequate to model convective envelopes and atmosphere covering all major evolutionary phases. Here we present a first step in this direction, where we have selected as reference choice the ML2. Our aim is to study whether a solar calibration with the ML2 provides a value of $\alpha_{\text {MLT }}$ close to what is employed in WD studies, how this "new" solar model compares with a BV58 solar model, and how evolutionary tracks computed with the solar calibrated ML2 compare with the BV58 calibration and with empirical determinations of $T_{\text {eff }}$ for Red Giant Branch (RGB) stars in Galactic globular clusters. Based on the results by Gough \& Weiss (1976) and Pedersen et al. (1990) we should expect that stellar models computed with different choices for $a, b$ and $c$ are largely equivalent once $\alpha_{\mathrm{MLT}}$ is appropriately recalibrated (although this has not yet been verified along the upper RGB) but we definitely do not know whether the same value of $\alpha_{\text {MLT }}$ employed in WD model atmosphere studies is adequate also for stellar modelling with the ML2.
Table 1. Values of the free parameters (besides $\alpha_{\mathrm{MLT}}$ ) in the BV58 and ML2 flavours of the MLT.

\begin{tabular}{lrrr}
\hline \hline MLT choice & $a$ & $b$ & $c$ \\
\hline BV58 & $\frac{1}{8}$ & $\frac{1}{2}$ & 24 \\
ML2 & 1 & 2 & 16 \\
\hline
\end{tabular}

We wish to stress that the aim of this note is not to argue for the superiority of the MLT over alternative and less crude prescriptions for stellar convection (see, e.g. Canuto \& Mazzitelli 1991), rather to investigate whether, within the framework of the MLT - that in spite of all its intrinsic limitations is still widely used in stellar evolution and model atmosphere calculations and with state-of-the-art physics inputs, it is possible to find a single combination of parameters suitable for computing interior and atmosphere models of all types of stars with superadiabatic convective layers. With "suitable" we mean that its use produces $T_{\text {eff }}$, spectral energy distributions and line profiles consistent with observations. The precise numerical value of $\alpha_{\text {MLT }}$ we derive for a given MLT flavour, is tied to the specific set of input physics adopted in the model computation. We employ what we believe are among the best possible physically motivated choices for these inputs. Inaccuracies in the current generation of inputs for stellar models (and model atmosphere) may of course impact the calibration of $\alpha_{\text {MLT }}$.

Section 2 summarizes the MLT formalisms and the choices of $a, b$ and $c$ in its ML2 and BV58 flavours. A comparison of solar stellar models, low mass stars in the metal poor regime and intermediate mass models computed with these two prescriptions for convection is discussed in Sect. 3, together with the comparison with $T_{\text {eff }}$ estimates of RGB stars in globular clusters. Conclusions follow in Sect. 4.

\section{MLT formalisms}

As already mentioned in the Introduction, there are three free parameters entering the MLT equations (denoted here as $a, b, c$ following the formalism of Tassoul et al. 1990) besides the mixing length. These 3 parameters appear (together with $\alpha_{\mathrm{MLT}}$ ) in the following equations for $v_{\mathrm{c}}, F_{\mathrm{c}}$ and $\Gamma$

$v_{\mathrm{c}}^{2}=\frac{a l^{2} g Q\left(\nabla-\nabla^{\prime}\right)}{H_{\mathrm{p}}}$

$F_{\mathrm{c}}=\frac{b \rho v_{\mathrm{c}} c_{\mathrm{p}} T l\left(\nabla-\nabla^{\prime}\right)}{H_{\mathrm{p}}}$

$\Gamma \equiv \frac{\nabla-\nabla^{\prime}}{\nabla^{\prime}-\nabla_{\mathrm{ad}}}=\frac{c_{\mathrm{p}} \rho^{2} l v_{\mathrm{c}} \kappa}{c \sigma T^{3}}$

where $\nabla^{\prime}$ is the temperature gradient of a rising (or falling) element of matter within the convective region, $\nabla$ is the average temperature gradient of all the matter at a given level within the convective zone (the quantity needed to solve the stellar structure equations $)$ and $Q \equiv-(\mathrm{d} \ln \rho / \mathrm{d} \ln T)_{P}$. Table 1 summarizes the BV58 and ML2 choices for $a, b, c$. From these expressions for $v_{\mathrm{c}}, F_{\mathrm{c}}$ and $\Gamma$ it is possible to obtain a simple algebraic equation whose solution provides the value of $\nabla$ at a given value of $r$.

Cox \& Giuli (1968) provides a widely used implementation of the BV58 flavour of the MLT. Here we show how to transform their implementation to describe the ML2. 
The equation for the effective temperature gradient in Cox \& Giuli (1968) is

$\zeta^{1 / 3}+B \zeta^{2 / 3}+a_{0} B^{2} \zeta-a_{0} B^{2}=0$

where $\zeta$ is defined as

$\zeta \equiv \frac{\nabla_{\mathrm{r}}-\nabla}{\nabla_{\mathrm{r}}-\nabla_{\mathrm{ad}}}$

Once the value of $\zeta$ is computed, the knowledge of $\nabla_{\mathrm{r}}$ (radiative gradient) and $\nabla_{\mathrm{ad}}$ (adiabatic gradient) provides immediately the actual gradient $\nabla$. The quantities $B$ and $a_{0}$ entering Eq. (4) are obtained through the following relationships:

$\Gamma=A\left(\nabla-\nabla^{\prime}\right)^{1 / 2}$

$\nabla_{\mathrm{r}}-\nabla=a_{0} A\left(\nabla-\nabla^{\prime}\right)^{3 / 2}$

$B \equiv\left[\left(A^{2} / a_{0}\right)\left(\nabla_{\mathrm{r}}-\nabla_{\mathrm{ad}}\right)\right]^{1 / 3}$.

To employ Eq. (4) with the ML2 choices of the constants $a, b$ and $c$ (Eqs. (1) $-(3)$ ), the quantities $a_{0}, A$, and $B$ calculated with the BV58 have to be transformed as follows:

$a_{0}(\mathrm{ML} 2)=a_{0}(\mathrm{BV} 58) \frac{8}{3}$

$A(\mathrm{ML} 2)=A(\mathrm{BV} 58) 3 \sqrt{2}$

$B(\mathrm{ML} 2)=B(\mathrm{BV} 58)\left(\frac{27}{4}\right)^{1 / 3}$

\section{Model comparison}

The canonical way of calibrating $\alpha_{\mathrm{MLT}}$ (together with the initial solar $\mathrm{H}, \mathrm{He}$ and metal mass fractions $X, Y$ and $Z$ ) is to compute a theoretical solar model that reproduces - at the solar age - the observed solar radius, luminosity and $(Z / X)_{\odot}$ ratio (see, e.g. Gough \& Weiss 1976). We have calibrated a theoretical solar model (including atomic diffusion) as described in Pietrinferni et al. (2004), starting from the pre-Main Sequence stage. Our adopted stellar evolution code and state-of-the-art input physics are fully described in Pietrinferni et al. (2004, and references therein) with the exception of the low temperature opacities $\left(T \leq 10^{4} \mathrm{~K}\right)$, for which we used here the updated calculations by Ferguson et al. (2005) that supersede the older Alexander \& Ferguson (1994) tables. The electron conduction opacities have also been updated, by employing the new calculations by Cassisi et al. (2007), although they are not important for the solar calibration. Other relevant physics inputs are the equation of state by Irwin ${ }^{1}$ that reproduces very well the results of the accurate OPAL (Rogers \& Nayfonov 2002) equation of state, as discussed in Pietrinferni et al. (2004); the OPAL radiative opacities (Iglesias \& Rogers 1996) for $T>10^{4} \mathrm{~K}$, and the NACRE nuclear reaction rates (Angulo et al. 1999). Atomic diffusion (without the effect of radiative levitation) is treated according to Thoul et al. (1994) and the surface boundary conditions were obtained by integrating the solar $T(\tau)$ relationship by Krishna Swamy (1966). We employed, again as in Pietrinferni et al. (2004), the Grevesse \& Noels (1993) solar metal mixture, given the strong conflict (Bahcall et al. 2005) between inferences

\footnotetext{
1 http://freeeos.sourceforge.net/
}

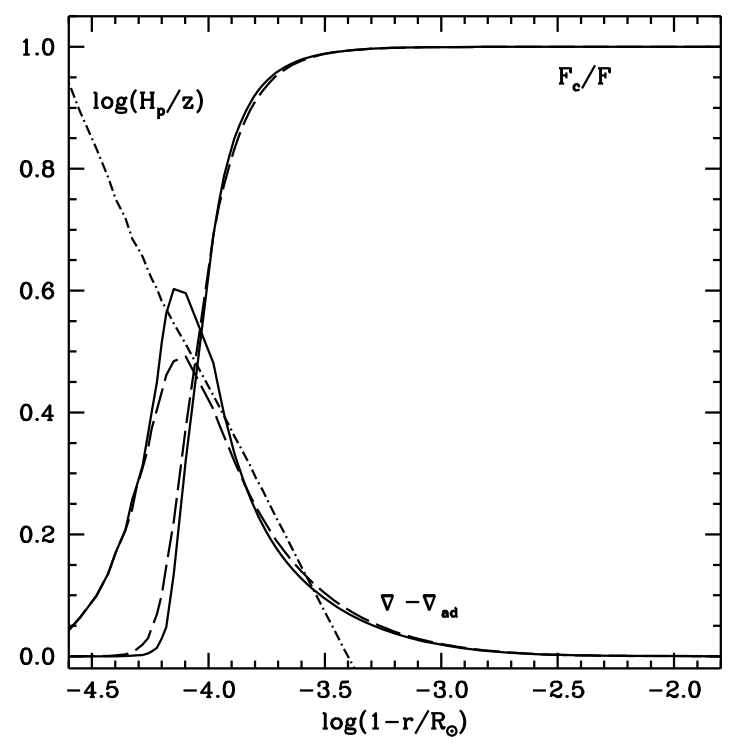

Fig. 1. Run of the superadiabaticity $\left(\nabla-\nabla_{\text {ad }}\right)$ and the ratio of the convective to the total energy flux as a function of the radial location in the outer layers of the solar convection zone. Solid lines represent the ML2 model, dashed lines the BV58 model. The dashed dotted line displays the ratio between the local pressure scale height and the geometrical distance from the top of the convective region.

from helioseismology and solar models computed with the recent Asplund et al. (2005) results.

The solar calibration with the BV58 and ML2 provides initial abundances $Y=0.2755$ and $Z=0.0201$ in both cases. The actual radius of the bottom of the convective envelope and He mass fraction in the envelope were the same in both calibrations, namely $R_{\mathrm{cz}}=0.7158 R_{\odot}$ and $Y=0.243$. The predicted value of $Z / X$ for the present Sun is $(Z / X)=0.0244$ in both calibrations, in agreement with $(Z / X)_{\odot}=0.0245 \pm 0.005$ by Grevesse \& Noels (1993). The calibrated values of $\alpha_{\mathrm{MLT}}$ are, respectively, $\alpha_{\odot, \mathrm{BV} 58}=$ 2.01 and $\alpha_{\odot, \mathrm{ML} 2}=0.63$. Notice the consistency of $\alpha_{\odot, \mathrm{ML} 2}$ with the best choice for WD atmosphere modelling.

To study the properties of superadiabatic regions calculated with the ML2, we compare in the following the run of some physical variables along the solar convective envelope, with both the BV58 and ML2 models. The difference $\left(\nabla-\nabla_{\mathrm{ad}}\right)$ in the outer layers is displayed in Fig. 1, and shows how the peak of the superadiabaticity is higher and slightly narrower with the ML2 formulation. This stems from the fact that at the location of the $\left(\nabla-\nabla_{\mathrm{ad}}\right)$ peak, the fraction of the total energy flux carried by convection is smaller in the ML2 model (see Fig. 1) hence a larger superadiabatic gradient is needed to satisfy the energy transport equation. The ratio between $F_{\mathrm{c}}$ and the total energy flux $F$ is also displayed and, as expected, differences between the two models are generally small and obviously restricted to the superadiabatic region. An interesting quantity also shown in Fig. 1 is the ratio between the local pressure scale height and the geometrical distance from the top of the convective envelope. Around the peak of the superadiabatic region the local value of $H_{\mathrm{p}}$ is about 3-3.5 times the distance from the surface. This highlights a well known inconsistency (e.g. Mazzitelli 1999) when using the BV58 calibrated values of $\alpha_{\mathrm{MLT}}$, given that with $\alpha \sim 2$, the mixing length $l$ in the superadiabatic region is about 6-7 times longer than the distance $z$ from the surface. Use of the ML2 alleviates - but does not eliminate - this conflict, producing a local $l$ more comparable with $z$. 


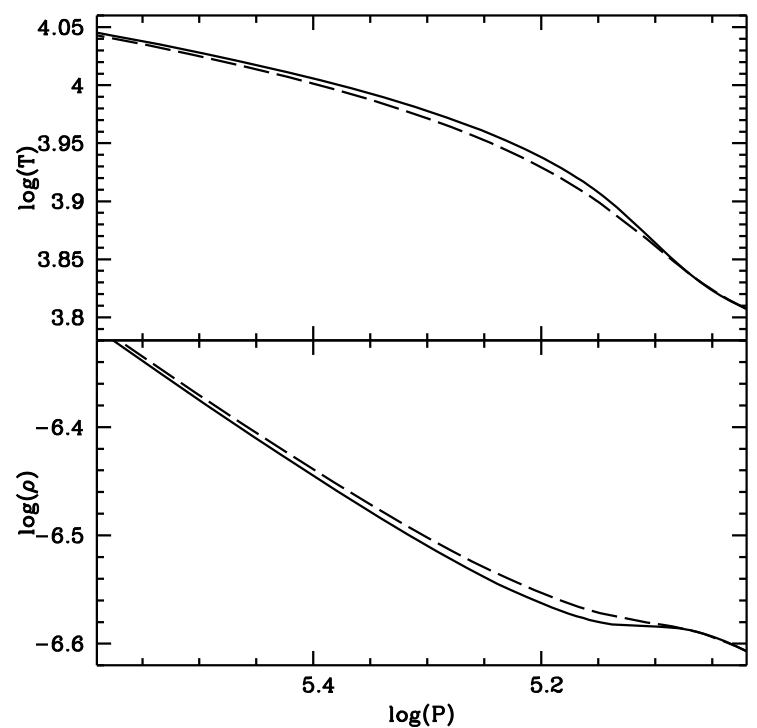

Fig. 2. Run of $T$ vs. $P$ (upper panel) and $\rho$ vs. $P$ (lower panel) in the outer layers of our solar models. The ML2 results are displayed as solid lines, the BV58 results as dashed lines.

The internal $P-T$ and $P-\rho$ stratifications are displayed in Fig. 2. Differences in the treatment of the superadiabatic layers produce slight modifications in the temperature and density values at a given $P$, that disappear when moving either towards the surface or towards the inner adiabatic layers. The largest differences in the stratification appear between $\log (P) \sim 5.10$ and $\log (P) \sim 5.15$, close to the region of the superadiabatic peak. Here, the temperature in the ML2 model increases more steeply towards the interior than in the BV58 case, and the $\rho$ profile is almost flat. Finally, Fig. 3 shows the relative sound speed $\left(v_{\mathrm{s}}=\sqrt{\frac{P \Gamma_{1}}{\rho}}\right)$ difference along the entire structure of the two models. The values of $v_{\mathrm{s}}$ are essentially identical except in the superadiabatic region (Fig. 1) as expected. However, the maximum difference is only $\sim 0.4 \%$ (the ML2 sound speed is larger) just below the location of the peak of the superadiabatic gradient. Moving inwards the ML2 sound speed becomes smaller by $0.2 \%$ and then equals the BV58 profile.

After calibrating the solar model we have computed a number of metal poor, low-mass models, from the pre-Main Sequence to the tip of the RGB, to compare solar calibrated $\alpha_{\text {MLT }}$ models in a regime different from the solar envelope and test predicted RGB $T_{\text {eff }}$ values against the determinations by Frogel et al. (1983) in a sample of Galactic globular clusters. Globular cluster RGB $T_{\text {eff }}$ estimates are one of the key data to test the treatment of superadiabatic convection in metal-poor low-mass stars (see, e.g. Salaris et al. 2002; Ferraro et al. 2006, and references therein).

To this purpose, stellar masses with a lifetime of 12-12.5 Gyr have been chosen as representative of objects populating the Turn Off and RGB of the observed Colour Magnitude Diagrams of Galactic globular clusters. We considered the $\alpha$ enhanced $([\alpha / \mathrm{Fe}]=0.4)$ metal mixture and $Y-Z$ relationship in the Pietrinferni et al. (2006) $\alpha$-enhanced models, and computed models for $[\mathrm{Fe} / \mathrm{H}]=-2.14,-1.62,-1.01,-0.60$, corresponding to $Z=0.0003,0.001,0.004$ and 0.01 , respectively. Sources for the input physics are the same as for the solar calibration. The boundary conditions for these models are still obtained by integrating the Krishna Swamy (1966) solar $T(\tau)$. The analysis by VandenBerg et al. (2008) has shown that boundary

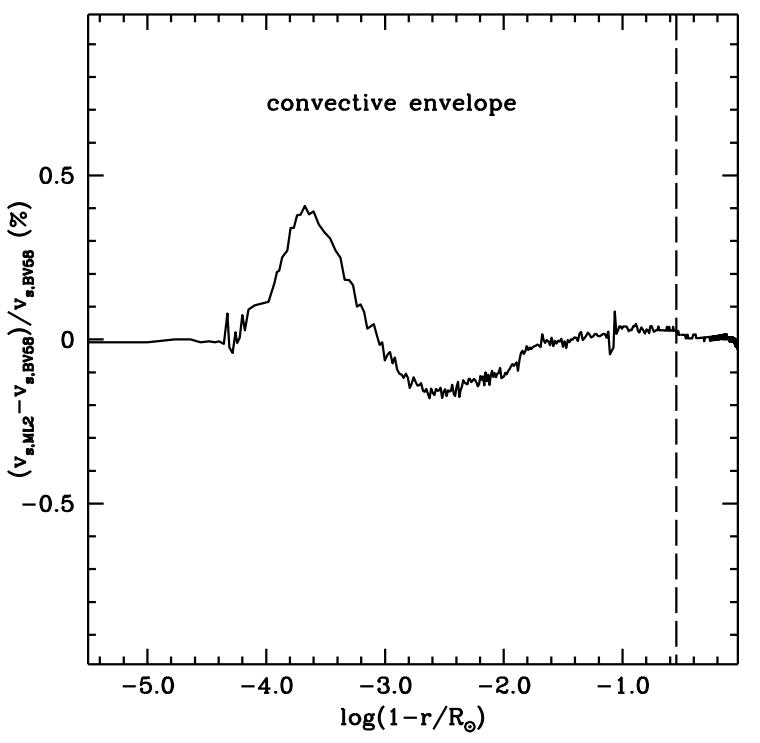

Fig. 3. Relative sound speed difference between the ML2 and BV58 models, as a function of the distance from the centre. The bottom of the convective region is marked by a vertical dashed line.

conditions from their differentially corrected model atmospheres (see VandenBerg et al. 2008, for details) that employ the same MLT constants as in the stellar interiors, provide almost the same solar $\alpha_{\text {MLT }}$ and also very similar $T_{\text {eff }}$ along the RGB at low metallicities as when the Krishna Swamy (1966) $T(\tau)$ relationship is used.

Figure 4 displays the tracks for $[\mathrm{Fe} / \mathrm{H}]=-2.14(M=$ $\left.0.8 M_{\odot}\right)$ and $[\mathrm{Fe} / \mathrm{H}]=-0.60\left(M=0.92 M_{\odot}\right)$ from the pre-Main Sequence to the tip of the RGB. The ML2 and BV58 tracks at the same $[\mathrm{Fe} / \mathrm{H}]$ are almost identical. Differences in $T_{\text {eff }}$ between the two sets of tracks begin to appear at the base of the RGB and slowly increase towards the RGB tip, when the superadiabatic region is located at lower temperatures and lower densities compared to previous evolutionary phases. In quantitative terms, they amount at most to $\sim 40 \mathrm{~K}$ for $[\mathrm{Fe} / \mathrm{H}]=-2.14$, and $\sim 50 \mathrm{~K}$ for $[\mathrm{Fe} / \mathrm{H}]=-0.60$. The luminosity of the RGB bump is the same, irrespectively of the MLT flavour, and also the amount of $\mathrm{He}$ dredged up along the RGB is the same. This is a consequence of the agreement between the location (in mass) of the bottom boundary of the surface convective regions in both sets of models, all the way to the tip of the RGB. Evolutionary timescales are unaffected by the change of the MLT flavour.

Figure 5 compares also estimates of the mean $T_{\text {eff }}$ at $M_{\text {bol }}=$ -3.0 for the RGBs of a sample of Galactic globular clusters by Frogel et al. (1983), with the corresponding quantities from our models. Theoretical bolometric luminosities have been transformed into bolometric magnitudes assuming $M_{\mathrm{bol}, \odot}=4.75$, to have the same zero point of Frogel et al. (1983) bolometric magnitudes. The cluster $[\mathrm{Fe} / \mathrm{H}]$ values are on the Carretta \& Gratton (1997) scale. We assigned an 0.1 dex error bar to the cluster $[\mathrm{Fe} / \mathrm{H}]$ values, and a $\pm 100 \mathrm{~K}$ error to the temperatures (as an estimate of realistic systematic errors in the RGB temperature scale, see e.g. Alonso et al. 1999).

Both MLT flavours produce $T_{\text {eff }}$ values that appear consistent with Frogel et al. (1983) data. We computed the differences between the predicted and estimated $T_{\text {eff }}$ for each cluster, by interpolating (with a cubic spline) among our results for the individual cluster metallicites. We did not find any statistical significant correlation between the $T_{\text {eff }}$ differences (observations-theory) and the corresponding $[\mathrm{Fe} / \mathrm{H}]$, for both ML2 and BV58 models. 


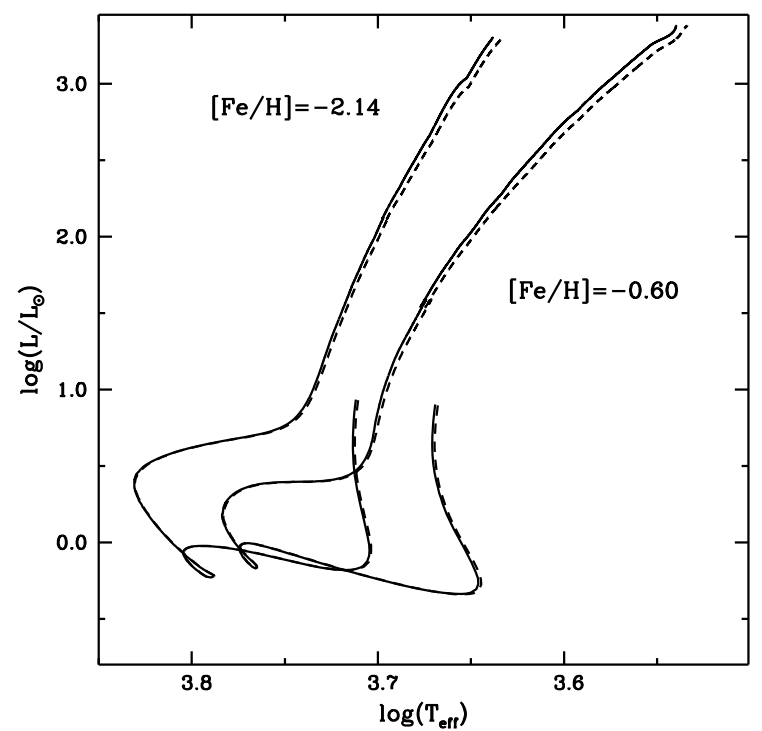

Fig. 4. Evolutionary tracks of low-mass models for the labelled $[\mathrm{Fe} / \mathrm{H}]$ values and an $\alpha$-enhanced metal distribution, from the pre-Main Sequence to the tip of the RGB, computed with the solar calibrated BV58 (dashed lines) and ML2 (solid lines) flavours of the MLT (see text for details).

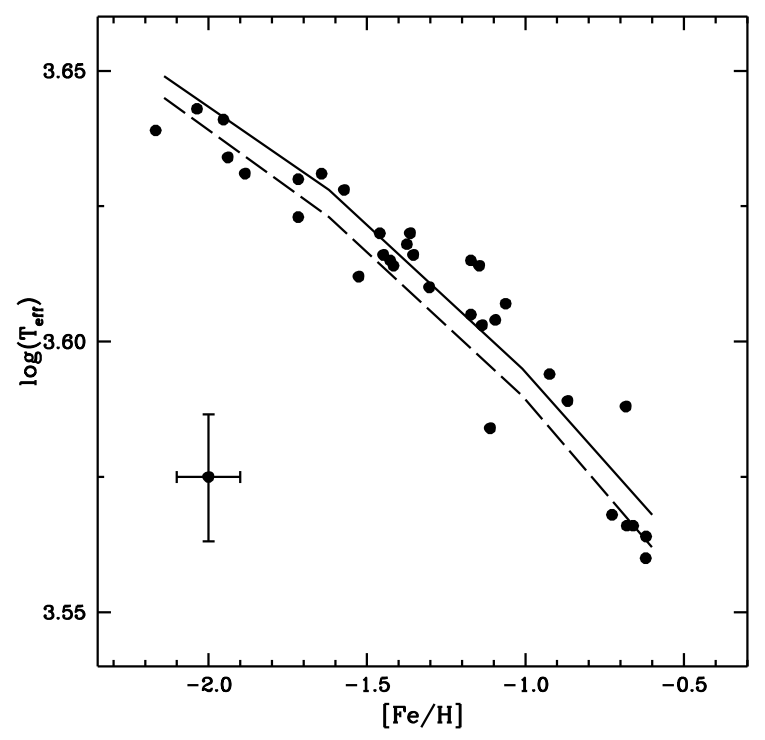

Fig. 5. Estimates of the average RGB $T_{\text {eff }}$ at $M_{\text {bol }}=-3$ for a sample of Galactic globular clusters, compared to the corresponding values from our models, computed with the solar calibrated BV58 (dashed line) and ML2 (solid line) flavours of the MLT. The assumed typical error bars are displayed in the lower left corner (see text for details).

In case of the ML2 models the average difference (observationstheory) is equal to $-15 \mathrm{~K}$, with a rms of $65 \mathrm{~K}$. The same difference for the BV 58 models is equal to $+32 \mathrm{~K}$, with a rms of $65 \mathrm{~K}$. The agreement with observations appear good in both cases. The smallest difference is for the ML2 results, but both sets of models provide $T_{\text {eff }}$ consistent with Frogel et al. (1983) if one considers the rms dispersion around this mean values and the estimated systematic errors on the RGB $T_{\text {eff }}$ scale.

We remark here that these models, at variance with the solar calibration, have been computed without including the effect of atomic diffusion, whose efficiency in Galactic globular cluster stars is still debated (Gratton et al. 2001; Korn et al. 2006). In any case, the efficiency of diffusion does not affect differential
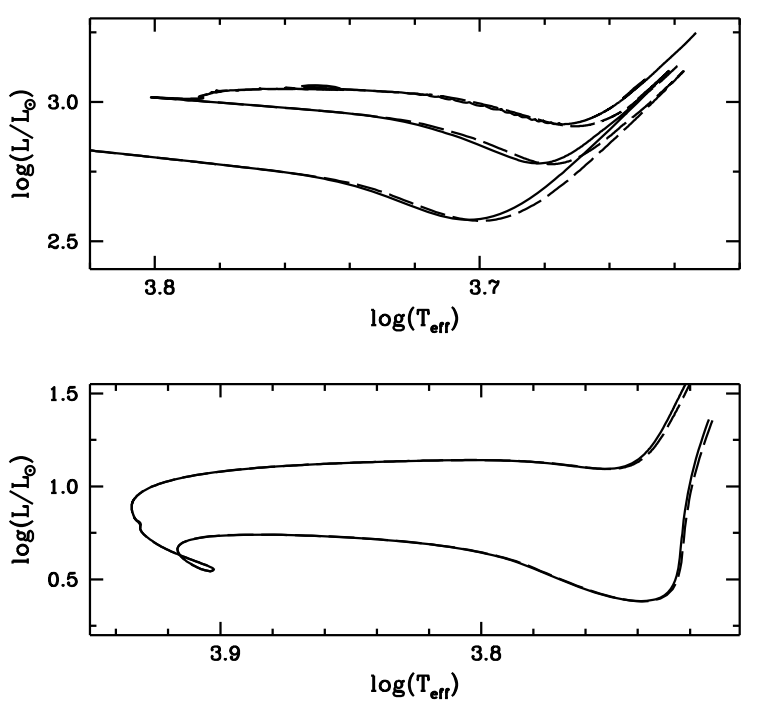

Fig. 6. Evolutionary tracks for, respectively, a $5 M_{\odot}$ star with solar chemical composition (from the subgiant branch to the early Asymptotic Giant Branch phase - upper panel) and a $1.2 M_{\odot}$ star with $[\alpha / \mathrm{Fe}]=0.4$ and $[\mathrm{Fe} / \mathrm{H}]=-1.62$ (from the pre-Main Sequence to the base of the RGB - lower panel). As in the previous figures, solid lines display the ML2 models, dashed lines the BV58 models.

comparisons along the Main Sequence, because of the same mass extension of convective envelopes in both ML2 and BV58 models (hence the same rate of depletion of surface $\mathrm{He}$ and metals in both cases, and the same effect on $\left.T_{\text {eff }}\right)$. Also, the comparison with estimated $T_{\text {eff }}$ values of RGB stars is robust, given that the location of the RGB in the H-R diagram is largely insensitive to the efficiency of diffusion.

As a final test, we display in Fig. 6 models for a $1.2 M_{\odot}$ star with an $\alpha$-enhanced, $Z=0.001([\mathrm{Fe} / \mathrm{H}]=-1.62)$ metal composition, and a $5 M_{\odot}$ star with solar composition, with both solar calibrated BV58 and ML2 convection. Both objects display during their evolution shallow or vanishing surface convective regions, and allow us to compare the two MLT flavours in the regime of very thin convective envelopes. The ML2 and BV58 calibrations produce again identical tracks along the Main Sequence and turn off region of the $1.2 M_{\odot}$ star, where convection is efficient in a very shallow outer layer. Differences in $T_{\text {eff }}$ along the pre-Main Sequence and RGB are as for the lower mass objects discussed above. Evolutionary tracks with the BV58 and ML2 for the $5 M_{\odot}$ solar composition model are also shown in Fig. 6. We have displayed the post-Main Sequence evolutionary phases where surface convection appears in the models. Differences in $T_{\text {eff }}$ are again small, reaching at most $50 \mathrm{~K}$ along the bright RGB and the early Asymptotic Giant Branch. The amount of $\mathrm{He}$ dredged up during the post-Main Sequence phases is the same with both treatments of surface convection, as a consequence of equal depths of the convective regions.

\section{Conclusions}

In the previous section we have shown that solar calibrated BV58 and ML2 stellar models computed with the input physics and boundary conditions described in Sect. 3, are very similar. There are some differences in the physical structures of the superadiabatic layers - as expected - together with minor variations in $T_{\text {eff }}$, but the extension of the convective regions are the same. Effective temperature differences appear essentially at the RGB and slowly increase towards the RGB tip, reaching at most 
$40-50 \mathrm{~K}$ at the lowest temperatures. This latter result does not conform exactly to Pedersen et al. (1990) conclusion that models computed with different choices for the MLT parameters $a$, $b$ and $c$ should be equivalent once $\alpha_{\mathrm{MLT}}$ is recalibrated, but the differences in the RGB $T_{\text {eff }}$ are not large.

Both ML2 and BV58 solar calibrated models provide a good match to $T_{\text {eff }}$ estimates for bright RGB stars in Galactic globular clusters. ML2 models appear to perform marginally better when observation-theory differences for each cluster are compared, but the effect of changing the MLT flavour is very small compared to both the rms scatter of the individual values, and realistic estimates of systematic errors in the RGB $T_{\text {eff }}$ scale.

In solar models, we have compared in some detail the sound speed, $T, \rho$ stratifications in the convective layers. If the ML2 improves the agreement between predicted and observed helioseismological properties (e.g. through the comparison of the solar $p$ mode excitation rates, see Samadi et al. 2006) is a matter to be established. On the other hand, due to its crudeness, we do not expect the MLT to be the adequate tool to model local details of superadiabatic convection to the degree of accuracy needed to satisfy helioseismological constraints on the structure of the solar superadiabatic layers.

A crucial point is that $\alpha_{\mathrm{MLT}}$ in the ML2 solar calibration obtained with our code and the adopted state-of-the-art stellar input physics is equal to 0.63 , a value consistent with the calibration preferred in WD model atmosphere calculations, that deal with a very different regime of gas properties. The exact value of the solar $\alpha_{\text {MLT }}$ may surely be subject to some variations, when different sources for the input physics are employed, but we expect that with nowadays best (i.e. most accurate and updated) possible choices, values around $\alpha_{\text {MLT }} \sim 0.6$ should still be found.

To verify whether this unification of the MLT treatment can be pushed even further, it will be necessary - as a next step to compute and test model atmospheres (plus spectra and bolometric corrections) for the pre-WD phases with surface convection, using the ML2 formalism with $\alpha_{\text {MLT }} \sim 0.6$. These ML2based model atmospheres, when computed and as a further test of this unified MLT treatment, should then be also employed to determine the boundary conditions of interior models, for studying the consistency with results obtained with a (solar) $T(\tau)$ integration.

Acknowledgements. S.C. acknowledges the financial support of INAF through the PRIN 2007 grant n. CRA 1.06.10.04 "The local route to galaxy formation: tracing the relics of the hierarchical merging process in the Milky Way and in other nearby galaxies". We warmly thank S. Degl'Innocenti for interesting discussions on the Solar Standard Model and Helioseismological constraints, and our referee (A. Dotter) for a prompt report and comments/suggestions that helped to improve the presentation of our results.

\section{References}

Alexander, D. R., \& Ferguson, J. W. 1994, ApJ, 437, 879

Alonso, A., Arribas, S., \& Martínez-Roger, C. 1999, A\&AS, 139, 335

Angulo, C., Arnould, M., Rayet, M., et al. 1999, Nucl. Phys. A, 656, 3

Asplund, M., Grevesse, N., \& Sauval, A. J. 2005, in Cosmic Abundances as Records of Stellar Evolution and Nucleosynthesis, ed. T. G. Barnes, III, \& F. N. Bash, ASP Conf. Ser., 336, 25

Bahcall, J. N., Basu, S., Pinsonneault, M., \& Serenelli, A. M. 2005, ApJ, 618, 1049

Bahcall, J. N., Serenelli, A. M., \& Basu, S. 2006, ApJS, 165, 400

Bergeron, P., Wesemael, F., \& Beauchamp, A. 1995a, PASP, 107, 1047

Bergeron, P., Wesemael, F., Lamontagne, R., et al. 1995b, ApJ, 449, 258

Biermann, L. 1932, Z. Astrophys., 5, 117

Böhm-Vitense, E. 1958, Zeitschrift fur Astrophysik, 46, 108

Brott, I., \& Hauschildt, P. H. 2005, in The Three-Dimensional Universe with Gaia, ed. C. Turon, K. S. O'Flaherty, \& M. A. C. Perryman, ESA SP-576, 565

Canuto, V. M., \& Mazzitelli, I. 1991, ApJ, 370, 295

Carretta, E., \& Gratton, R. G. 1997, A\&AS, 121, 95

Cassisi, S., Potekhin, A. Y., Pietrinferni, A., Catelan, M., \& Salaris, M. 2007, ApJ, 661, 1094

Castelli, F., Gratton, R. G., \& Kurucz, R. L. 1997, A\&A, 318, 841

Cox, J. P., \& Giuli, R. T. 1968, Principles of stellar structure (New York: Gordon and Breach)

Dotter, A., Chaboyer, B., Jevremović, D., et al. 2007, AJ, 134, 376

Ferguson, J. W., Alexander, D. R., Allard, F., et al. 2005, ApJ, 623, 585

Ferraro, F. R., Valenti, E., Straniero, O., \& Origlia, L. 2006, ApJ, 642, 225

Fontaine, G., Brassard, P., \& Bergeron, P. 2001, PASP, 113, 409

Frogel, J. A., Cohen, J. G., \& Persson, S. E. 1983, ApJ, 275, 773

Girardi, L., Bressan, A., Bertelli, G., \& Chiosi, C. 2000, A\&AS, 141, 371

Gough, D. O., \& Weiss, N. O. 1976, MNRAS, 176, 589

Gratton, R. G., Bonifacio, P., Bragaglia, A., et al. 2001, A\&A, 369, 87

Grevesse, N., \& Noels, A. 1993, in Origin and Evolution of the Elements, ed. S. Kubono, \& T. Kajino, 14

Gustafsson, B., Edvardsson, B., Eriksson, K., et al. 2008, A\&A, 486, 951

Heiter, U., Kupka, F., van't Veer-Menneret, C., et al. 2002, A\&A, 392, 619

Henyey, L., Vardya, M. S., \& Bodenheimer, P. 1965, ApJ, 142, 841

Iglesias, C. A., \& Rogers, F. J. 1996, ApJ, 464, 943

Korn, A. J., Grundahl, F., Richard, O., et al. 2006, Nature, 442, 657

Krishna Swamy, K. S. 1966, ApJ, 145, 174

Mazzitelli, I. 1999, in Stellar Structure: Theory and Test of Connective Energy

Transport, ed. A. Gimenez, E. F. Guinan, \& B. Montesinos, ASP Conf. Ser., 173, 77

Morel, P., \& Lebreton, Y. 2008, ArXiv e-prints, 801

Pedersen, B. B., Vandenberg, D. A., \& Irwin, A. W. 1990, ApJ, 352, 279

Pietrinferni, A., Cassisi, S., Salaris, M., \& Castelli, F. 2004, ApJ, 612, 168

Pietrinferni, A., Cassisi, S., Salaris, M., \& Castelli, F. 2006, ApJ, 642, 797

Rogers, F. J., \& Nayfonov, A. 2002, ApJ, 576, 1064

Rohrmann, R. D. 2001, MNRAS, 323, 699

Salaris, M., Cassisi, S., \& Weiss, A. 2002, PASP, 114, 375

Samadi, R., Kupka, F., Goupil, M. J., Lebreton, Y., \& van't Veer-Menneret, C. 2006, A\&A, 445, 233

Tassoul, M., Fontaine, G., \& Winget, D. E. 1990, ApJS, 72, 335

Thoul, A. A., Bahcall, J. N., \& Loeb, A. 1994, ApJ, 421, 828

VandenBerg, D. A., Swenson, F. J., Rogers, F. J., Iglesias, C. A., \& Alexander, D. R. 2000, ApJ, 532, 430

VandenBerg, D. A., Edvardsson, B., Eriksson, K., \& Gustafsson, B. 2008, ApJ, 675,746

Yi, S., Demarque, P., Kim, Y.-C., et al. 2001, ApJS, 136, 417 